Revue française de pédagogie

띰 PÉDAGOGIE

\author{
Recherches en éducation
}

192 | juillet-août-septembre 2015

Face aux mutations des marchés de l'emploi, quelles

politiques de formation?

\title{
Mondialisation, progrès technique et dépréciation du capital humain. L'impact sur les politiques de formation
}

Globalisation, technological change, and human capital depreciation. The impact on education policy

Sylvain Weber, Giovanni Ferro Luzzi et José V. Ramirez

\section{(2) OpenEdition}

Journals

Édition électronique

URL : http://journals.openedition.org/rfp/4816

DOI : 10.4000/rfp.4816

ISSN : 2105-2913

Éditeur

ENS Éditions

Édition imprimée

Date de publication : 30 septembre 2015

Pagination : 11-22

ISSN : 0556-7807

Référence électronique

Sylvain Weber, Giovanni Ferro Luzzi et José V. Ramirez, « Mondialisation, progrès technique et dépréciation du capital humain. L'impact sur les politiques de formation », Revue française de pédagogie [En ligne], 192 I juillet-août-septembre 2015, mis en ligne le 30 septembre 2018, consulté le 02 janvier 2020. URL : http://journals.openedition.org/rfp/4816 ; DOI : 10.4000/rfp.4816 


\title{
Mondialisation, progrès technique et dépréciation du capital humain L'impact sur les politiques de formation
}

\author{
Sylvain Weber \\ Giovanni Ferro Luzzi \\ José V. Ramirez
}

\begin{abstract}
Le capital humain et les politiques visant à sa création et à sa préservation prennent de plus en plus d'importance dans les sociétés industrialisées. Cet article propose un survol de la littérature économique récente dans ce domaine. Le défi majeur qui occupe aujourd'hui le marché du travail est l'accélération du progrès technique, qui s'accompagne d'effets différenciés suivant le niveau de qualification des travailleurs. Deux théories sont évoquées pour analyser ces effets. D'une part, le progrès technique est vu comme biaisé en faveur des travailleurs qualifiés et défavorable aux moins qualifiés. D'autre part, et suite au phénomène de polarisation du marché du travail récemment observé, on peut cependant penser que la technologie est surtout substituable aux emplois du milieu de l'échelle. De ces constatations découlent plusieurs recommandations de politiques économiques, la principale étant de donner aux travailleurs les moyens d'être flexibles et polyvalents, ce qui passe par une éducation relativement générale plutôt que trop spécifique et cantonnée à un secteur ou une profession.
\end{abstract}

Mots-clés (TESE) : capital humain, système éducatif, marché du travail, politique en matière d'éducation, mondialisation.

\section{Introduction}

Cette contribution vise à documenter la recherche récente en économie sur le capital humain et les chocs subis par ce dernier, qui modifient parfois très substan- tiellement les relations d'offre et de demande de qualifications. Les chocs considérés sont la croissance des échanges internationaux, la plus grande mobilité des facteurs productifs qui l'accompagne et surtout le progrès technique. Isoler ces perturbations et mesurer 
leurs effets sur l'emploi et les salaires est en soi ardu, mais les modifications des politiques (salaires minimums, systèmes éducatifs, sécurité sociale...) et de l'offre de travail des ménages brouillent encore davantage la lisibilité quant à l'impact de ces chocs.

Une partie de la littérature économique a tenté de montrer l'effet prépondérant du progrès technique sur le rendement du capital humain et les inégalités de salaires qui en ont résulté. Des travaux plus récents ont cependant montré que l'impact du progrès technique, mais aussi des délocalisations, était plus subtil que la simple amélioration des salaires des travailleurs qualifiés par rapport aux non-qualifiés. II semble en réalité qu'il s'agisse surtout de certains métiers et tâches caractérisés par une forte routine et répétition qui ont souffert et pas nécessairement les emplois peu qualifiés. Cela pose bien entendu des questions en matière de politique de formation. Comment former pour mieux protéger les travailleurs contre le risque de dépréciation de leur capital humain? Dans quelle mesure faut-il privilégier la formation générale au détriment de la formation spécifique? Faudrait-il introduire plus de formation spécifique au niveau secondaire, comme dans les pays caractérisés par un système de formation duale comme l'Allemagne ou la Suisse? Ou, au contraire, introduire plus de formation générale dans la formation professionnelle? La question est loin d'être simple. En effet, la spécificité du capital humain a longtemps été considérée comme une protection contre les revers de conjoncture. II semble cependant que, face à une certaine volatilité de la demande de savoirs et savoir-faire, une formation générale assure plus que jamais une meilleure protection contre les aléas du progrès technique ou des possibles délocalisations qui touchent certains secteurs.

Le reste de cet article est organisé comme suit. La première partie présente les différents types de dépréciation susceptibles d'affecter le capital humain. La deuxième se concentre sur les effets du progrès technique sur le capital humain, en soulignant notamment le biais de la technologie en faveur du travail qualifié. La troisième partie décrit le phénomène de polarisation des emplois mis en évidence récemment dans les pays industrialisés. Des réflexions relatives aux politiques de formation pouvant permettre de contrer la dépréciation du capital humain sont proposées dans la dernière partie.

\section{Le capital humain et sa dépréciation}

La théorie du capital humain, développée par Becker (1964), part de l'idée que l'éducation permet d'acquérir des compétences qui améliorent la productivité du travailleur ${ }^{1}$. Puisque la productivité est supposée déterminer le salaire, un travailleur ayant accumulé plus de capital humain obtiendra un salaire plus élevé. Le temps passé à se former est donc considéré comme un investissement (éducatif) qui implique des coûts immédiats suivis de bénéfices obtenus sous forme de revenus supérieurs pendant la carrière professionnelle. Dans son modèle original, Becker (1964) considère que le capital humain peut être catégorisé comme général, c'est-à-dire valorisé de manière identique dans toutes les entreprises, ou comme spécifique, c'est-à-dire utile dans une seule et unique entreprise. Ces deux types de capital humain sont le plus souvent mêlés dans la formation (en particulier la formation en emploi), celle-ci n'étant ni complètement spécifique ni complètement générale. Ultérieurement, la littérature a proposé des extensions contenant des types de capital humain «intermédiaires» (voir par exemple Sullivan, 2010). II semble en effet plausible qu'une partie du capital humain puisse être spécifique à une profession ou une industrie, sans toutefois être complètement général ou spécifique. La frontière entre le capital humain spécifique et le capital humain général ainsi que leur substituabilité ou complémentarité dans la production de valeur restent floues et ouvertes à débat.

Une fois acquis, le capital humain est susceptible de se déprécier, qu'il soit spécifique ou général, et ce sous l'effet de plusieurs forces. Le tableau 1, inspiré de De Grip \& Van Loo (2002), donne un aperçu détaillé des différents types de dépréciation. La dépréciation interne (également appelée obsolescence technique) concerne les éléments attribuables au travailleur luimême. L'usure est ainsi causée par des facteurs biologiques. En vieillissant, le travailleur va inéluctablement oublier certaines de ses connaissances et ses capacités physiques et mentales vont s'amenuiser. D'éventuelles maladies ou accidents pourraient également avoir un

1 Les compétences se définissent comme la mobilisation des ressources que sont les connaissances (savoir), les habiletés (savoirfaire) et les attitudes (savoir-être). Pour une discussion plus approfondie sur les définitions des compétences, voir par exemple Dujardin (2013, chap. 2). 


\begin{tabular}{ll}
\hline \multicolumn{1}{c}{ Type de dépréciation } & \multicolumn{1}{c}{ Causes } \\
\hline $\begin{array}{l}\text { Dépréciation interne } \\
\text { Usure }\end{array}$ & Vieillissement naturel, maladies ou blessures \\
Atrophie & Non-utilisation ou sous-utilisation \\
Dépréciation externe & \\
Au niveau de l'emploi & Changements technologiques ou organisationnels \\
Au niveau de l'entreprise & Mobilité (volontaire ou involontaire) \\
Au niveau macroéconomique & Réduction de la demande de travail dans une industrie
\end{tabular}

effet néfaste. D'autre part, un individu inactif ou chômeur n'utilisera pas pleinement son potentiel et risque alors une atrophie de son capital humain. Le travailleur en situation de suréducation est confronté à une problématique similaire.

La dépréciation externe (également appelée obsolescence économique) provient quant à elle de l'environnement du travailleur. Des changements nuisibles au capital humain peuvent en effet se produire au niveau de l'emploi, au niveau de l'entreprise ou au niveau macroéconomique. Par exemple, lorsqu'une entreprise décide de réorganiser sa structure, certains travailleurs seront repositionnés, voire licenciés. Ces travailleurs n'effectueront alors plus exactement les mêmes tâches qu'auparavant : certaines de leurs connaissances ne seront plus utiles et ils devront en acquérir de nouvelles. Autrement dit, une partie plus ou moins importante de leur capital humain deviendra inutile suite à la réorganisation de l'entreprise. Des innovations technologiques peuvent avoir des effets similaires. L'exemple typique est l'arrivée des ordinateurs, qui a rendu obsolète la quasi-totalité des connaissances permettant l'utilisation d'une machine à écrire.

Il est intéressant de souligner que des changements au niveau du système éducatif lui-même peuvent engendrer de la dépréciation. En effet, lorsque les matières enseignées sont modifiées, sous l'effet du progrès technique et donc pour les rapprocher des besoins du marché du travail, les travailleurs déjà en place se retrouvent avec un capital humain moins adapté que celui des nouvelles générations. Ce phénomène est connu sous le terme d'effets de millésime (Neuman \& Weiss, 1995 ; Ramirez, 2002).

Comme mentionné auparavant, une partie du capital humain est supposée être spécifique à l'entreprise dans laquelle le travailleur est actif. Au cas où un travailleur quitte son emploi, la part de capital humain qui était spécifique à son ancienne entreprise devient inutile. Ceci démontre qu'une mobilité accrue sur le marché du travail (volontaire ou non) risque d'accroître la vitesse de dépréciation. À ce propos, il est utile de préciser que, même si le sentiment populaire semble indiquer une augmentation de la mobilité professionnelle, les études statistiques ne parviennent que difficilement à confirmer cette impression (cf. Weber \& Ferro Luzzi, 2014).

Des changements de plus grande échelle peuvent également produire de la dépréciation externe, par exemple en réduisant la demande de travail au niveau d'une industrie voire d'un secteur économique. On observe ce type de changements lorsque des entreprises décident de délocaliser voire de cesser leurs activités sous l'effet d'une intensification de la compétition internationale dans leur secteur.

Il est important de souligner que progrès technique et mondialisation s'accompagnent nécessairement de dépréciation externe. En effet, toute innovation rendra obsolètes certaines compétences des travailleurs utilisant une technologie ancienne et vouée à disparaître. De même, en raison de l'intensification des échanges internationaux, la délocalisation d'activités par les entreprises devient plus fréquente, ce qui engendre la disparition d'emplois voire de pans entiers de l'économie. Les travailleurs concernés subissent alors inévitablement un préjudice puisqu'ils doivent trouver un nouvel emploi dans lequel certains de leurs savoirs et savoir-faire ne seront pas valorisés.

Il faut remarquer que les différents types de dépréciation ne sont pas mutuellement exclusifs. Ainsi, tout travailleur subira de la dépréciation interne sous l'effet de l'usure, à laquelle pourrait s'ajouter de la dépréciation externe subie suite à un licenciement. En réalité, 
il semble même plausible que les différents types de dépréciation s'influencent mutuellement. Par exemple, on peut logiquement supposer qu'un travailleur fortement sujet à l'usure, c'est-à-dire qui aurait tendance à oublier des connaissances, sera soumis à un risque de licenciement accru. À l'inverse, un travailleur licencié qui tombe au chômage subira non seulement de la dépréciation externe mais également une atrophie de son capital humain.

Le cas du progrès technique est intéressant dans le contexte de la relation entre les différents types de dépréciation. Considérons un secteur économique dans lequel les innovations sont fréquentes et graduelles. Les travailleurs de ce secteur seront naturellement amenés à renouveler leurs compétences régulièrement. La dépréciation externe qu'ils subissent aurait donc tendance à être forte, mais les actions de ces travailleurs tendront à la contrer et on peut alors s'attendre à ce que leur dépréciation interne soit faible. En revanche, dans un secteur habituellement stable, si une innovation inattendue se produit, cela pourrait décourager certains travailleurs qui ne feront pas l'effort de s'adapter et devront peut-être changer d'emploi voire quitter le marché du travail de façon anticipée. Bartel et Sicherman (1993) montrent en effet que les décisions de départ à la retraite sont influencées par le rythme du progrès technique.

Plusieurs contributions discutent les effets attendus de l'éducation (ou plus précisément du nombre d'années d'éducation) sur la dépréciation du capital humain. Neuman et Weiss (1995) supposent que la dépréciation augmente avec le niveau d'éducation puisque le capital humain acquis en école élémentaire ne souffre pas d'obsolescence, étant donné que le programme d'enseignement n'évolue guère dans le temps à ce niveau. Cependant, plusieurs raisons laissent penser que l'éducation pourrait freiner la dépréciation. Weisbrod (1962) note qu'une personne plus éduquée aura plus de facilité à s'adapter aux changements. On remarque effectivement que les individus initialement plus éduqués suivent par la suite plus régulièrement des formations additionnelles. Cipriani (1967) remarque également que diplômes et certificats fonctionnent comme des «tickets d'admission » pour d'autres formations. Une formation plus générale permettrait également de réduire le coût d'adaptation du capital humain aux nouveaux besoins de savoir et savoir-faire créés par les chocs technologiques (Stankiewicz, 1995). Par ailleurs, on peut relever que les modèles de demande pour la santé (par exemple
Grossman, 2000) font l'hypothèse que l'éducation réduit la dépréciation du «stock de santé » (qui fait partie intégrante du stock du capital humain). Un individu plus éduqué aurait en effet tendance à ménager son stock de capital humain grâce à son style de vie, ses décisions quant à la nourriture et à l'activité physique, ou encore grâce à une meilleure utilisation de l'information médicale dans la production de santé.

Au niveau empirique, les résultats sur le lien entre durée d'éducation et dépréciation sont peu nombreux et mitigés. Mincer et Polacheck (1974) et Rosen (1976) obtiennent des taux de dépréciation plus élevés pour les travailleurs plus éduqués aux États-Unis. Murillo (2011) obtient un résultat similaire pour l'Espagne. Au contraire, Rosen (1975) trouve une dépréciation plus faible pour les travailleurs américains sortant du college (niveau tertiaire) par rapport à ceux n'ayant obtenu qu'un diplôme high school (niveau secondaire II). Finalement, Arrazola, De Hevia, Risueno et alii (2005) ne trouvent pas de différences significatives entre les travailleurs espagnols différemment formés, et Groot (1998) obtient des résultats contradictoires en utilisant une approche identique pour le Royaume-Uni et les Pays-Bas.

Le fait que la littérature se soit focalisée sur le lien entre durée d'éducation et dépréciation s'explique sans doute parce qu'elle est fortement orientée sur le cas des États-Unis, où le système éducatif est «linéaire» et la formation professionnelle très peu développée. On peut cependant imaginer que la vitesse de dépréciation dépende du type de capital humain dont il est question. En particulier, on peut s'attendre à ce que le capital humain général se déprécie moins facilement sous l'effet de la dépréciation externe que le capital humain spécifique. Dès lors, si le type d'éducation peut être relié à un type de capital humain, on doit s'attendre à ce que les individus provenant de branches différentes du système éducatif subissent des taux de dépréciation différents.

Dans la littérature allant dans ce sens, Krueger et Kumar (2004) soutiennent que la propension à utiliser la formation professionnelle plutôt que générale pourrait être une cause de la différence entre les taux de croissance américain et européen. Leur argument est que la formation professionnelle, qui apprend à l'étudiant à effectuer une tâche particulière, par opposition à la formation générale, qui permet à l'étudiant de maîtriser un concept, conduit à une adoption moins rapide des nouvelles technologies. Hanushek, Schwerdt, Woessmann et alii (à paraître) avancent éga- 
lement que les compétences générées par l'enseignement professionnel facilitent sans doute la transition vers le marché du travail, mais peuvent par la suite devenir obsolètes à un rythme plus rapide. À partir de données sur la Suisse, où la formation professionnelle (en entreprise) est largement répandue, Weber (2014) obtient un taux de dépréciation plus élevé pour les travailleurs avec formation professionnelle par rapport à ceux avec formation académique. Ces résultats semblent donc indiquer que le type d'éducation, plus que sa durée, exerce une influence sur la dépréciation du capital humain.

Il faut également souligner qu'il n'est pas évident de dire si c'est l'éducation reçue en elle-même qui affecte la dépréciation ou si les différences observées sont dues à des effets de sélection. Le fait que les travailleurs issus des branches professionnelles suivent significativement moins de formations en cours de carrière suggère qu'une partie des différences est due à des caractéristiques individuelles (telles qu'un goût variable pour les études) ou aux caractéristiques des emplois occupés. La formation professionnelle a ainsi parfois été considérée comme une impasse éducationnelle (Ryan, 2001), notamment dans les pays caractérisés par un système éducatif «classique», où la formation duale au niveau du secondaire reste encore marginale comparativement à des pays ayant une forte culture de l'apprentissage en entreprise. Globalement, les résultats empiriques tendent donc à montrer qu'une formation à plus forte composante générale permet au travailleur d'évoluer plus facilement dans sa carrière (en termes de salaire, d'emploi, de dépréciation...), et ce indépendamment de la durée des études.

\section{Les biais de la technologie}

De nombreuses recherches menées essentiellement aux États-Unis aboutissent à un consensus concernant l'accroissement des inégalités salariales en fonction des qualifications (voir par exemple Juhn, Murphy \& Pierce, 1993). Les causes sous-jacentes évoquées pour expliquer cette montée des inégalités sont en revanche nettement moins évidentes. En effet, la période commençant dans les années 1980 a été marquée par la conjonction de trois phénomènes importants : la mondialisation progressive avec un accroissement notable des échanges internationaux, l'irruption des nouvelles technologies de l'information et de la communication (TIC) à tous les échelons de l'activité économique et enfin, dans nombre de pays dévelop- pés, les modifications plus structurelles liées au marché du travail (telles que les variations du salaire minimum, l'érosion du pouvoir des syndicats et l'entrée en vigueur d'assurances contre les risques du chômage) qui ont toutes pu affecter la distribution des salaires et, partant, le rendement des différents types de qualifications ainsi que les incitations à acquérir ces dernières. Cette partie se penche sur les bouleversements qu'ont pu provoquer les innovations technologiques sur le marché du travail.

Dans leur ouvrage judicieusement intitulé La course entre éducation et technologie, Goldin et Katz (2008) décrivent le xxe siècle comme le «siècle du capital humain » car c'est durant cette période que l'éducation publique s'est le plus étendue et a permis d'accroître la proportion de travailleurs qualifiés. Le phénomène a d'abord été observé aux États-Unis, puis en Europe avec des différences substantielles entre pays. Les auteurs examinent la relation entre cette forte progression de main-d'œuvre éduquée et la compression concomitante des écarts salariaux jusqu'à la fin de la seconde guerre mondiale. L'offre de travailleurs avec un niveau bac ou universitaire ayant augmenté régulièrement pendant ces années, les salaires du haut de la distribution ont subi une forte modération de leur croissance. Par la suite, dans les années 1950, les écarts de salaires s'accroissent jusqu'environ au début des années 1970 où ils se réduisent brièvement avant de s'envoler à partir des années 1980. Le xxe siècle a également connu des changements technologiques majeurs déjà avant la première guerre mondiale, de sorte qu'il n'est pas évident d'imputer les changements récents de manière univoque à la simple apparition des TIC.

Quelles sont dès lors les conséquences de l'intégration quasi généralisée des TIC, et en particulier des ordinateurs, dans le système de production? Les auteurs s'accordent pour considérer que les ordinateurs ont notablement affecté les processus de production et partant la distribution des salaires entre professions, mais également au sein des professions. Autor, Katz et Krueger (1998) montrent par exemple que la revalorisation des compétences (via l'engagement de travailleurs plus qualifiés) a été nettement plus marquée dans les secteurs plus intensifs en ordinateurs, même s'ils admettent que leurs résultats ne permettent pas d'en déduire une causalité. DiNardo \& Pischke (1997) mettent d'ailleurs en garde sur l'interprétation de résultats montrant des primes salariales pour les employés faisant usage d'ordinateurs en raison de l'évident biais de sélection : les personnes en contact avec les ordinateurs ne sont pas seulement plus qualifiées de manière formelle 
mais disposent de compétences inobservables qui sont recherchées par les employeurs et rémunérées en conséquence. Un ordinateur peut faciliter certaines tâches, en particulier celles qui sont routinières et répétitives. Toutefois, la littérature semble indiquer que l'introduction des ordinateurs a considérablement modifié l'organisation de la production et du travail dans l'entreprise (décentralisation, dé-hiérarchisation, déspécialisation), ce qui engendre des coûts allant bien au-delà de l'achat des ordinateurs et qui peut amener à demander des nouvelles compétences sans que celles-ci soient directement en lien avec I'utilisation d'ordinateurs, comme la capacité d'adaptation à des situations de plus en plus changeantes au poste de travail (Stankiewicz, 1995). Deming (2015) discute également l'importance croissante des compétences sociales suite aux progrès des TIC, qui ont modifié l'organisation du travail en rendant les structures plus flexibles et autogérées, et les travailleurs plus multitâches.

On ne peut donc pas résumer l'impact de la révolution numérique à un simple accroissement d'informaticiens ou d'ingénieurs système. La plus grande complémentarité s'observe avec des travailleurs ayant des qualifications élevées (typiquement de niveau d'éducation tertiaire), mais aussi de grandes compétences «soft » et «humaines » telles que l'interaction humaine, la créativité, et bien entendu la résolution de problèmes complexes, en bref tout ce qui permet une maîtrise des tâches non routinières et que les ordinateurs ne parviennent pas encore à remplacer. Le changement technologique provoque ainsi des changements dans les tâches requises et induit une réassignation des travailleurs demandés dans les tâches en fonction de leurs avantages comparatifs. Autor, Levy et Murnane (2003) montrent que les secteurs d'activité caractérisés par une prépondérance des tâches routinières sont ceux ayant le plus massivement investi dans l'équipement informatique à partir des années 1970 et qui ont subséquemment réduit leur demande de travailleurs non qualifiés et augmenté celle de travailleurs qualifiés.

Il convient enfin de mentionner le changement de paradigme dans l'appréhension du progrès technique et de ses effets sur les qualifications. Simplement affirmer que le changement technologique "cause» une demande accrue de travailleurs qualifiés ne dit rien sur la source de ce changement technologique. Acemoglu (2002) en veut pour preuve que le changement technologique avec biais envers les qualifications a commencé avant la seconde guerre mondiale alors que les inégalités salariales ne se sont accrues que dans les trente dernières années $d u x^{e}$ siècle, ce qui ne serait cohérent qu'à condition que l'offre de travailleurs qualifiés ait ralenti sur cette dernière période, ce qui n'est bien entendu pas le cas. L'explication la plus plausible et cohérente avec un accroissement des inégalités couplé à une augmentation de l'offre de travailleurs qualifiés reste donc du côté d'une accélération du progrès technique sur la fin du $x x^{e}$ siècle.

Le progrès technique doit donc être vu comme endogène aux chocs qui affectent l'offre de maind'œuvre dans les différentes professions et leur niveau de qualifications. Ainsi, l'augmentation de l'offre de travail non qualifié via l'exode rural au xix ${ }^{\mathrm{e}}$ siècle aurait induit l'adoption de technologies nouvelles permettant leur absorption dans le processus de production et remplaçant des artisans et autres travailleurs qualifiés ou semi-qualifiés par des machines et des ouvriers non qualifiés. Un phénomène semblable est à l'œuvre dans la fin du xx siècle : l'accroissement de l'offre de travailleurs qualifiés permet I'adoption des TIC, de sorte que les employeurs peuvent engager une masse critique de travailleurs qualifiés et réarranger rentablement leur processus de production grâce notamment au moindre coût d'adaptation de leur main-d'œuvre. Dans une étude sur des données d'entreprises industrielles françaises, Duguet et Greenan (1997) montrent qu'effectivement l'innovation se caractérise par un biais technologique en faveur du travail qualifié, mais également que l'innovation est endogène et déterminée par la proportion de personnel de conception.

Quelle que soit la réponse politique apportée au biais de l'innovation technologique, on ne peut guère exclure qu'une partie des travailleurs se retrouvent durablement exclus du monde du travail en raison de qualifications insuffisantes et que la demande pour des tâches simples, répétitives et routinières se réduise dans les pays occidentaux.

\section{La polarisation des emplois}

II subsiste peu de doute quant au fait que les TIC exercent un fort impact sur le marché du travail. Cependant, I'hypothèse du biais technologique en faveur des emplois qualifiés a récemment été contestée et décrite comme n'expliquant qu'une partie de la vérité. Cette hypothèse ne permet effectivement pas d'expliquer certains changements importants du marché du travail, et notamment la polarisation des emplois (Card \& DiNardo [2002] listent d'autres phénomènes inexpliqués). II semble en effet que la distribution des emplois ait tendance à se 
déformer dans les pays industrialisés, avec une érosion des emplois de «milieu de gamme», ainsi définis sur la base de leur rémunération (et donc du niveau de qualification). Au contraire, les emplois versant les salaires les plus élevés ainsi que ceux versant les salaires les plus faibles gagnent en nombre. On se retrouve alors avec une distribution des emplois polarisée, avec des extrêmes renforcés (voir par exemple Goos \& Manning, 2007, pour le Royaume-Uni ; Autor, Katz \& Kearney, 2006, pour les États-Unis; Goos, Manning \& Salomon, 2009 pour l'Europe; Oesch \& Rodríguez Menés, 2011, pour I'Angleterre, I'Allemagne, I'Espagne et la Suisse).

Les causes de la polarisation mises en évidence par la littérature sont principalement liées à la routinisation des tâches (Autor, Levy \& Murnane, 2003) et à la globalisation (Blinder, 2007). Les tâches routinières dans lesquelles les TIC peuvent potentiellement remplacer le travail humain sont effectuées dans des emplois tels que l'artisanat ou la comptabilité, qui exigent un certain niveau de qualification et qui par conséquent ne sont pas les moins rémunérés, mais qui impliquent la réalisation de tâches répétitives. En revanche, les tâches non routinières complémentaires aux nouvelles technologies sont réalisées dans des emplois qualifiés qui nécessitent généralement des compétences cognitives non routinières (gestion, conception, création, développement, coordination ou organisation) et qui se situent en général dans la partie supérieure de la distribution des salaires. Les tâches manuelles non routinières, qui composent la plupart des emplois moins qualifiés tels que le nettoyage ou les services aux personnes, ne sont pas directement touchées par les TIC. Cependant, sous l'effet des TIC, l'évolution générale de l'économie est susceptible de conduire à une augmentation de ces emplois. Dans ce cas, la technologie cause une hausse de la demande relative des emplois qualifiés et bien rémunérés et des emplois les moins qualifiés et faiblement rémunérés. À l'inverse, la technologie cause une baisse de la demande relative dans les emplois qui nécessitent généralement une combinaison plus ou moins équilibrée de capacités manuelles et cognitives, et qui se trouvent dans le milieu de l'échelle salariale.

L'intensification des échanges internationaux semble également jouer un rôle dans la polarisation des emplois. D'une part, les emplois de «milieu de gamme», tels que la plupart des postes de production dans l'industrie, semblent être les plus fortement soumis à la concurrence internationale, puisque ce sont précisément ces tâches qui peuvent être facilement délocali- sées. D’autre part, les emplois du haut de l'échelle salariale voient leur nombre augmenter, car ils bénéficient de l'augmentation des échanges internationaux, qui accroît notamment les besoins de gestion. Enfin, les emplois les moins rémunérés, tels que les services aux personnes, ne peuvent que difficilement, voire pas du tout être délocalisés. Ces emplois ont également tendance à devenir plus nombreux en raison de l'évolution démographique des pays industrialisés et des changements dans les préférences des ménages (notamment les plus qualifiés) quant à leur offre de travail.

Sur ce dernier point, Lindley et Machin (2013) ont observé, à partir de données américaines portant sur la période 1980 à 2010, que la concentration de travailleurs très qualifiés, qui s'observe notamment dans les grands centres urbains, est également en soi une source de polarisation des emplois. L'accroissement du salaire des personnes hautement qualifiées, et donc de leur coût d'opportunité à effectuer du travail ménager, les incite à participer plus activement au marché du travail. Les femmes ayant des enfants travaillent plus fréquemment qu'autrefois, en particulier lorsqu'elles ont reçu une formation d'un certain niveau. II en résulte que la demande pour les services domestiques, faiblement qualifiés et faiblement rémunérés, augmente. Les changements sociétaux plus profonds, comme la plus grande propension au divorce, accentuent cette corrélation entre la demande de travail très qualifié et celle pour des emplois "locaux», soumis à la concurrence internationale exclusivement par le biais de l'immigration.

Finalement, sur le lien entre polarisation des emplois et système éducatif, Rendall et Weiss (2015) remettent en question la corrélation dans le temps entre le poids des emplois associés aux tâches routinières et I'intensité d'utilisation des TIC. Elles observent en effet que les régions allemandes (Länder) qui se caractérisaient par une proportion relativement faible d'emplois de «milieu de gamme» à la fin des années 1970 sont celles qui aujourd'hui utilisent le plus intensivement les TIC, soit l'inverse de ce qui a pu être observé aux États-Unis. Ces différences entre États-Unis et Allemagne seraient avant tout le fruit du système éducatif, c'est-à-dire de l'importante de l'apprentissage en entreprise, par essence plus spécifique que la formation académique. Pour une entreprise formant des apprentis, l'acquisition d'une nouvelle machinerie est en effet plus coûteuse puisqu'elle rend obsolète la formation (spécifique) transmise via l'apprentissage. Autrement dit, la propension des entreprises allemandes à former 
des apprentis, et donc à accroître la composante spécifique de leur capital humain, freine leur incitation à investir dans des modèles de production plus intensifs en TIC. Ce frein prend encore davantage d'importance si on considère que les apprentis sont en moyenne plus productifs que les autres travailleurs semi-qualifiés, précisément grâce à leurs compétences plus spécifiques à l'entreprise.

\section{Quid des politiques éducatives?}

Le défi posé par la dépréciation du capital humain consiste à trouver des solutions pour la contrer, ou du moins en amortir les effets délétères sur la productivité. Identifier correctement la cause de la dépréciation est crucial puisque le remède en dépend. Dans le cas d'une dépréciation due à l'usure ou à l'atrophie, le travailleur aura besoin essentiellement d'une remise à niveau, simplement pour se remémorer des notions déjà acquises par le passé. Si en revanche la dépréciation est externe au niveau de l'emploi ou de l'entreprise, le travailleur aura besoin de ressources supplémentaires afin de complémenter celles qu'il possède déjà. La formation continue devrait l'aider à pouvoir évoluer dans sa carrière et, si nécessaire, à trouver un nouvel emploi similaire au précédent dans une autre entreprise. Enfin, si la dépréciation externe provient d'une réduction de la demande de travail au niveau d'un secteur d'activité, fournir des connaissances additionnelles propres à ce domaine ne sera d'aucun secours. Dans un tel cas de figure, les travailleurs auront surtout besoin de compétences nouvelles qu'ils ne possèdent pas encore.

Le point commun à toutes les solutions visant à contrer la dépréciation externe du capital humain est donc de rendre les travailleurs plus perméables à une accumulation plus fréquente de capital humain tout au long de leur carrière, soit au travers des requalifications soit par la poursuite de formation continue en cours d'emploi. Cette meilleure adaptabilité ou flexibilité - définie comme la capacité à effectuer des tâches qui ne font pas directement partie de son travail ${ }^{2}$ - constitue en effet l'assurance de pouvoir trouver un emploi

2 Van Loo, De Grip et De Steur (2001) utilisent le terme «flexibilité fonctionnelle » pour décrire ce concept. La Commission européenne (2007) a quant à elle introduit le terme "flexicurité » pour indiquer que flexibilité et sécurité (assurance chômage avec revenus de remplacements élevés, prise en charge proactive, formation, etc.) sont complémentaires et devraient donc être conjointement rapidement et dans une large gamme d'entreprises et de secteurs économiques.

Reste la question plus délicate de l'évolution de la distribution des salaires et des probabilités d'emploi. Quelle devrait être la réponse publique en regard non seulement de la moins grande équité du système qui en résulte mais également du sort des travailleurs les moins qualifiés, dont les perspectives semblent devenir plus sombres? Dans ce cas également, la formation continue tout au long du cycle de vie est souvent mentionnée mais les résultats semblent plutôt décevants, l'essentiel de cette formation étant le fait de travailleurs les plus qualifiés, les mieux dotés en capital humain général, qui en ont a priori le moins besoin. En Suisse, une enquête de l'Office fédéral de la statistique (OFS, 2010) révèle que, parmi les travailleurs sans formation post-obligatoire, moins d'un sur cinq a participé à une formation continue durant les 12 derniers mois. Parmi les personnes ayant une formation de niveau tertiaire, cette même proportion se monte à deux sur trois. Cette inégalité se creuse encore davantage si on ne considère que les formations pour raisons professionnelles : seul un travailleur sans formation post-obligatoire sur dix y prend part, contre un sur deux parmi les travailleurs avec formation tertiaire.

En amont des politiques de remédiation, I'encouragement de la formation professionnelle dite duale (dispensée non seulement en école mais également en partie en entreprise), axée sur le marché du travail, telle qu'elle est pratiquée en Suisse ou en Allemagne peut fournir une piste intéressante. Ainsi que nous l'avons vu, l'accélération du progrès technique incite les employeurs à modifier leur processus de production et à engager des travailleurs davantage en fonction de leurs compétences que de leurs qualifications. Or, la versatilité de cette demande implique que les employeurs soient partie prenante des formations à fournir, et la formation duale en entreprise permet justement aux travailleurs d'acquérir les compétences demandées en temps réel. De manière plus générale (cf. Lerman, 2013), les avantages d'une formation professionnelle duale sont le renforcement des connaissances théoriques par l'application dans le monde du travail, une participation rémunérée au secteur productif et bien entendu une meilleure transition de l'école au travail, puisque les personnes ont un contrat de travail pendant leur période d'apprentissage. En ajoutant que la formation duale exige moins de ressources publiques par rapport à d'autres stratégies de formation, il est compréhensible que cette voie de formation soit actuellement si prisée. 
Mais comme la formation continue, la formation professionnelle duale en entreprise ne constitue en aucun cas la panacée face aux changements qui affectent les économies occidentales. La polarisation des emplois constitue sans aucun doute une menace, puisque les individus détenteurs d'un diplôme de formation professionnelle sont pour la plupart actifs dans des emplois du milieu de l'échelle des qualifications. Ce constat met une fois encore en lumière les avantages et les risques de la formation professionnelle : d'un côté, lorsqu'elle est duale, une telle formation permet une intégration rapide au marché du travail, mais d'une autre côté elle protège relativement peu en cas de concurrence internationale accrue. Pour faire en sorte que la formation professionnelle duale puisse apporter davantage de bénéfices sur le long terme, il est important qu'un rééquilibrage en faveur de sa composante générale s'opère, afin de fournir à ses détenteurs une flexibilité accrue face aux changements de l'environnement de travail.

De manière générale, il semble aujourd'hui presque superflu de souligner l'accent qui doit être mis sur la maîtrise des outils de communication, qu'ils soient matériels (outils informatiques) ou immatériels (langages); mais également sur l'importance d'être capable d'interagir dans un monde devenu plus hétérogène, que ce soit par le biais de l'origine culturelle des individus, leur genre voire leur âge. Ces deux compétences, alliées à celle de l'autonomie, forment selon I'OCDE (2005) les trois compétences-clés nécessaires pour être en mesure de répondre aux défis que pose la société moderne et pour développer des compétences tout au long de la vie professionnelle. L'accumulation d'expériences dans des contextes différents permet dès lors à l'individu d'améliorer sa pratique réflexive, qui se situe au cœur même des compétencesclés et qui fait appel à des savoir-faire métacognitifs et à des facultés de création qui se développent tôt dans la vie, mais qui peuvent continuer de s'acquérir à tout âge. La qualité de l'apprentissage tout au long de la vie se joue probablement déjà avant l'âge de 6 ans, comme le suggèrent des résultats empiriques récents quant à l'effet de l'éducation préscolaire sur différents indicateurs en lien avec la scolarité et le marché du travail (Heckman, 2012). II n'est donc pas inutile de rappeler que les autorités publiques ne devraient pas seulement être attentives aux politiques dites de remédiation, comme les besoins de financement de la formation des adultes, ou à celles de la formation professionnelle en secondaire supérieur, mais qu'elles devraient tout autant se pencher sur le financement et l'organisation de l'éducation «préscolaire».

\section{Conclusion}

Ce bref survol de la littérature économique sur le capital humain démontre à quel point le rendement de I'investissement éducatif peut s'avérer sensible à des changements de l'environnement de travail, induits entre autres par l'innovation technologique. Les effets du progrès technique sont ainsi loin d'être univoques et dépendent crucialement de la relation de complémentarité ou de substituabilité qui s'établit avec les différents types de travailleurs.

L'hypothèse de biais technologique en faveur du travail qualifié suggère ainsi que les travailleurs qualifiés sont complémentaires au progrès technique et devraient par conséquent en bénéficier, à l'inverse des travailleurs moins qualifiés. La polarisation des emplois, observée dans la plupart des pays industrialisés, laisse cependant penser que si la technologie est effectivement complémentaire avec le travail exigeant un haut niveau de qualification, elle n'est en fait substituable qu'aux emplois situés dans le milieu de l'échelle salariale mais non avec les emplois les moins qualifiés et plus faiblement rémunérés. Ces derniers ne sont pas directement affectés par le progrès technique, mais leur nombre est indirectement poussé à la hausse en raison de l'évolution générale de l'économie.

Ces éléments suggèrent que des travailleurs de niveaux d'éducation différents et/ou situés à des niveaux différents de l'échelle salariale doivent s'attendre à subir une dépréciation du capital humain plus ou moins rapide. Les travailleurs ayant des qualifications les situant dans le milieu de l'échelle salariale, où le nombre d'emplois a tendance à diminuer, seront plus fréquemment forcés à changer de postes, ce qui exigera d'eux l'acquisition de compétences nouvelles. La dépréciation externe subie par ces travailleurs sera alors nécessairement plus forte que pour des travailleurs dont l'environnement est plus stable.

Quels enseignements tirer en termes de recommandations pour les systèmes éducatifs? De manière générale, une personne plus instruite aura plus de facilité à trouver un emploi, à suivre une formation continue, et à s'adapter à un nouvel environnement. Encourager les travailleurs à se former est donc la première recommandation à formuler, mais elle est déjà largement répandue. Il s'agit donc essentiellement de repérer les bonnes mesures incitatives. La question se 
pose cependant au-delà de la simple durée (ou quantité) d'éducation, bien que ce soit l'aspect le plus fréquemment étudié dans la littérature. Le contenu de l'éducation est effectivement un élément fondamental dans la carrière d'un individu. Ainsi, un travailleur ayant suivi une formation professionnelle aura sans doute un parcours très différent de celui qui aurait suivi une formation académique, et ce même si la durée d'éducation de ces deux travailleurs est absolument identique et que le domaine étudié est similaire.

Dans une période où le marché du travail devient plus instable, les atouts majeurs pour les travailleurs sont la flexibilité et la faculté d'adaptation. Un objectif important pour le système éducatif semble par conséquent résider dans l'équilibre entre capital humain général et spécifique. En particulier, il importe que chaque travailleur dispose d'un socle de capital humain général lui permettant de s'adapter à de nombreuses situations professionnelles. Les systèmes éducatifs allemand et suisse, dans lesquels la formation professionnelle en entreprise occupe une place prépondérante, offrent sur ces points des pistes de réflexion intéressantes. Les travailleurs issus de la formation

\section{Bibliographie}

ACEMOGLU A. (2002). «Technical change, inequality, and the labor market ». Journal of Economic Literature, $\mathrm{n}^{\circ} 40(1)$, p.7-72.

ARRAZOLA M., DE HEVIA J., RISUENO M. \& SANZ J. (2005). "A proposal to estimate human capital depreciation: Some evidence for Spain ». Hacienda Publica EspanolaRevista de Economia Publica, ${ }^{\circ}{ }^{172(1), ~ p . ~ 9-22 . ~}$

AUTOR D., KATZ L. \& KRUEGER A. (1998). «Inequality: Have computers changed the labor market?». The Quarterly Journal of Economics, $n^{\circ} 113(4)$, p. 1169-1213.

AUTOR D., LEVY F. \& MURNANE R. (2003). «The skill content of recent technological change: An empirical exploration ». Quarterly Journal of Economics, nº 118(4), p. 1279-1333.

AUTOR D., KATZ L. \& KEARNEY M. (2006). «The polarization of the US labor market». American Economic Review, $n^{\circ}$ 96(2), p.189-194.

BARTEL A. \& SICHERMAN N. (1993). «Technological change and retirement decisions of older workers ». Journal of Labor Economics, $\mathrm{n}^{\circ} 11(1)$, p. 162-183.

BECKER G. (1964). Human capital: A theoretical and empirical analysis with special reference to education. New York : National Bureau of Economic Research.

BLINDER A. (2007). "Offshoring: Big deal, or business as usual?». Center for Economic Policy Studies Working Paper, $\mathrm{n}^{\circ} 149$. professionnelle duale ont en effet plus de facilité à trouver un premier emploi. En cours de carrière, ils semblent cependant connaître moins de succès, avec un taux de dépréciation de leur capital humain plus élevé et une croissance moindre de leur rémunération. Étant donné la part relativement importante de capital humain spécifique inhérente à la formation professionnelle par rapport aux formations académiques, l'importance de fournir des compétences générales prend alors tout son sens.

Sylvain Weber

Université de Neuchâtel, Institut de recherches économiques sylvain.weber@unine.ch

Giovanni Ferro Luzzi

Université de Genève, Institut d'économie et d'économétrie et Haute école de gestion de Genève giovanni.ferro-luzzi@unige.ch

José V. Ramirez

Haute école de gestion de Genève et NCCR LIVES jose.ramirez@hesge.ch

CARD D. \& DINARDO J. (2002). «Skill-biased technological change and rising wage inequality: Some problems and puzzles ». Journal of Labor Economics, n²0(4), p.733-783.

CIPRIANI C. (1967). «Hedging in the labor market». Southern Economic Journal, n³4(2), p.286-292.

COMMISSION EUROPÉENNE (2007). Vers des principes communs de flexicurité : des emplois plus nombreux et de meilleure qualité en combinant flexibilité et sécurité. Bruxelles : Commission européenne.

DE GRIP A. \& VAN LOO J. (2002). "The economics of skills obsolescence: A review». In A. De Grip, J. Van Loo \& K. Mayhew (dir.), The Economics of Skills Obsolescence: Theoretical Innovations and Empirical Applications, Vol. 21 of Research in Labor Economics. Amsterdam : Elsevier Science, p.1-26.

DEMING D. J. (2015). «The growing importance of social skills in the labor market». National Bureau of Economic Research Working Paper, nº 21473.

DINARDO J. \& PISCHKE J.-S. (1997). «The returns to computer use revisited: Have pencils changed the wage structure too?». Quarterly Journal of Economics, n 112(1), p. 291-303.

DUJARDIN J.-M. (2013). Compétences durables et transférables. Clés pour l'employabilité. De Boeck : Bruxelles.

DUGUET E. \& GREENAN N. (1997). «Le biais technologique. Une analyse économétrique sur des données individuelles». Revue économique, n48(5), p. 1061-1089. 
GOLDIN C. \& KATZ L. (2008). The race between education and technology. Cambridge (États-Unis) : The Belknap Press of Harvard University Press.

GOOS M. \& MANNING A. (2007). «Lousy and lovely jobs: The rising polarization of work in Britain ». Review of Economics and Statistics, $n^{\circ} 89(1)$, p. 118-133.

GOOS M., MANNING A. \& SALOMONS A. (2009). «Job polarization in Europe». American Economic Review, n 99(2), p. 58-63.

GROOT W. (1998): «Empirical estimates of the rate of depreciation of education ». Applied Economics Letters, n5(8), p. 535-538.

GROSSMAN M. (2000). "The human capital model». In A. J. Culyer \& J.P. Newhouse (dir.), Handbook of Health Economics, vol. 1A. Amsterdam : Elsevier, p.347-408.

HANUSHEK É., SCHWERDT G., WOESSMANN L. \& ZHANG L. (à paraître). "General education, vocational education, and labor-market outcomes over the life-cycle». Journal of Human Resources.

HECKMAN J. (2012). Giving kids a fair chance. Cambridge (États-Unis) : MIT Press.

JUHN C., MURPHY K. \& PIERCE B. (1993). «Wage inequality and the rise in returns to skill ». Journal of Political ECOnomy, $\mathrm{n}^{\circ} 101(3)$, p. 410-442.

KRUEGER D. \& KUMAR K. (2004). «Skill-specific rather than general education: A reason for US-Europe growth differences? ». Journal of Economic Growth, n॰ 9(2), p. 167-207.

LERMAN R. (2013). «Skill development in middle level occupations: The role of apprenticeship training ». IZA Policy Paper, $\mathrm{n}^{\circ} 61$.

LINDLEY J. \& MACHIN S. (2013). «Spatial changes in labour market inequality». IZA Discussion Paper, $\mathrm{n}^{\circ} 7600$.

MINCER J. \& POLACHEK S. (1974). «Family investments in human capital: earnings of women ». Journal of Political Economy, n 82(2), p. S76-S108.

MURILLO I. (2011). «Human capital obsolescence: some evidence for Spain». International Journal of Manpower, $\mathrm{n}^{\circ} 32(4), \mathrm{p} .426-445$.

NEUMAN S. \& WEISS A. (1995). "On the effects of schooling vintage on experience-earnings profiles: Theory and evidence». European Economic Review, n³9(5), p. 943-955.

OCDE (2005). La définition et la sélection des compétences clés. Paris : OCDE.
OESCH D. \& RODRÍGUEZ MENÉS J. (2011). «Upgrading or polarization? Occupational change in Britain, Germany, Spain and Switzerland, 1990-2008». Socio-Economic Review, $\mathrm{n}^{\circ}$ 9, p. 503-531.

OFS (2010). Participation à la formation continue en Suisse. Berne : Office fédéral de la statistique.

RAMIREZ J. (2002). «Age and schooling vintage effects on earnings profiles in Switzerland ». In A. de Grip, J. Van Loo \& K. Mayhew (dir.), The Economics of Skills Obsolescence: Theoretical Innovations and Empirical Applications, vol.21 of Research in Labor Economics. Amsterdam : Elsevier Science, p. 83-99.

RENDALL M. \& WEISS F. (2015). «Employment Polarization and the Role of the Apprenticeship System ». University of Zürich, Working paper, $\mathrm{n}^{\circ} 313$.

ROSEN S. (1975). "Measuring the obsolescence of knowledge». In F. T. Juster (dir.), Education, Income and Human Behavior. New York : National Bureau of Economic Research, p.199-232.

ROSEN S. (1976). «A theory of life earnings ». Journal of Political Economy, n84(4), p. S45-S67.

RYAN P. (2001). «The school-to-work transition: A crossnational perspective». Journal of Economic Literature, n³9(1), p. 34-92.

STANKIEWICZ F. (1995). «Choix de formation et critères d'efficacité du travail ». Revue économique, n5, p. 1311-1331.

SULLIVAN P. (2010). «Empirical evidence on occupation and industry specific human capital». Labour Economics, $\mathrm{n}^{\circ} 17(3)$, p. 567-580

VAN LOO J., DE GRIP A. \& DE STEUR M. (2001). «Skills obsolescence: causes and cures». International Journal of Manpower, $\mathrm{n}^{\circ} 22(1 / 2)$, p. 121-138.

WEISBROD B. (1962). «Education and investment in human capital ». Journal of Political Economy, $n^{\circ}$ 70(5), p. 106-123.

WEBER S. (2014). «Human capital depreciation and education level». International Journal of Manpower, $n^{\circ} 35(5)$, p. 613-642.

WEBER S. \& FERRO LUZZI G. (2014). «From lifetime jobs to churning?». Swiss Journal of Economics and Statistics, $\mathrm{n}^{\circ} 150(3)$, p. $227-260$. 
